\title{
Towards gamma ray bursts localization with the BeppoSAX/GRBM
}

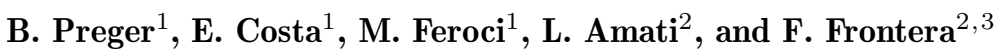 \\ 1 Istituto di Astrofisica Spaziale, C.N.R. Area di Ricerca Tor Vergata, Roma, Italy \\ 2 Istituto T.E.S.R.E., C.N.R., Bologna, Italy \\ 3 Dipartimento di Fisica, Universita' di Ferrara, Italy
}

Received March 6; accepted April 23, 1999

\begin{abstract}
The method to evaluate the capability to detect and localize Gamma Ray Bursts (GRBs) with the Gamma Ray Burst Monitor (GRBM) onboard BeppoSAX is presented. We have used a sample of GRBs simultaneously detected by the GRBM and the Burst And Transient Source Experiment (BATSE) onboard CGRO to characterize the dependence of the GRBM trigger efficiency on the intensity, hardness and incoming direction of GRBs and to compare the results of our method with known locations.
\end{abstract}

Key words: gamma-rays: bursts — instrumentation: detectors

\section{Introduction}

Non-imaging gamma-ray detectors can localize GRBs by using the relative flux registered with individual detectors oriented at different directions. This method is used, as an example, by the BATSE experiment (Fishman et al. 1985) and can also be used by the GRBM (Pamini et al. 1990) onboard BeppoSAX (Boella et al. 1997), if the response function of each detector is known with satisfactory accuracy, though larger error boxes are expected. However, even a rough determination of the angular position can be used to choose between the two intersections of annuli derived by the IPN, for bursts which BATSE does not detect. In order to test this method on GRBM, we have chosen a sample of GRBs simultaneously detected by GRBM and BATSE, for which the incoming direction and spectral characteristics can be determined from the BATSE catalog (http://www.batse.msfc.nasa.gov). In the following we briefly show a study of the BATSE/GRBM relative trigger efficiency, describe the method developed for GRB localization and give the preliminary results obtained for a typical event.

\section{The GRBM onboard BeppoSAX}

The Gamma Ray Burst Monitor (e.g. Frontera et al. 1997; Feroci et al. 1997) is composed of the four veto shields of the high energy PDS experiment, which is located in the inner part of the spacecraft (see e.g. Frontera et al. 1997;

Send offprint requests to: preger@saxserver1.ias.rm.cnr.it
Table 1. BATSE/GRBM relative trigger efficiency from May, $23^{\text {rd }} 1997$ to June, $6^{\text {th }} 1998$

\begin{tabular}{lr}
\hline Number of days & 374 \\
BATSE triggers & 322 \\
GRBM simultaneous triggers & 61 \\
GRBM hardware OFF & 119 \\
GRBM software OFF & 3 \\
Detected but not triggered & 24 \\
Data not available & 11 \\
\hline Common triggers & $32 \%$ \\
Common detections & $46 \%$ \\
\hline
\end{tabular}

Costa et al. 1998). This location causes a partial obscuration of the instrument's field of view by other parts of the satellite. Moreover, each detector is affected by different elements of the scientific payload, causing a different energy and direction dependence of the response for the four detectors. Detectors GRBM1 and GRBM3 are affected by the Low and Medium Energy Concentrator Spectrometers, GRBM2 by the HPGSPC and GRBM4 by the Wide Field Cameras. On-ground calibrations performed after the full integration of the spacecraft have been analized, corrected for environmental effects, interpolated and fitted with physical and semiempirical functions (Amati et al. 1997) to provide the response of each detector as a function of the incoming direction and the spectrum of GRBs. To evaluate the accuracy of this positioning we started an extended check of the response of GRBM to GRBs located by BATSE.

To test the capability of GRBM of detecting GRBs (Feroci et al. 1997), we have considered a sample of events simultaneosly detected by BATSE and GRBM and studied their characteristics of time duration, intensity and spectral properties. A deep study carried out over about one year has given the results listed in Table 1 where with "Hardware OFF" we indicate that the instrument is switched off, and this can occur for example during the crossing of the South Atlantic Geomagnetic Anomaly, or in case of satellite maintenaince. With "Software OFF", instead, we indicate that the instrument is on, but the software is not enabled: data are stored and can be analized, but the trigger function is inactive. A particular care was devoted to the study of possible selection effects on the GRBM trigger efficiency (see also Feroci et al. 1999), showing a clear selection on duration 


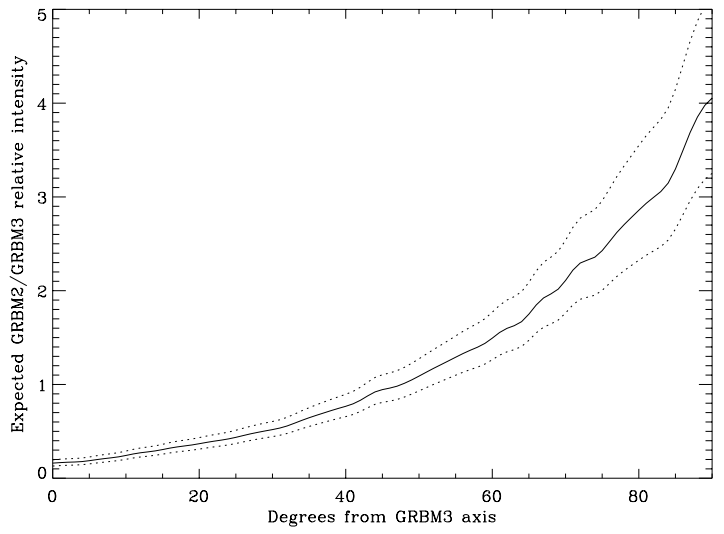

Fig. 1. Dependence of the expected GRBM2/GRBM3 relative intensity on the azimuthal angle $\phi$ with respect to the GRBM3 axis (computed for altitude $\theta=6.8^{\circ}$, spectral index $\alpha=0.9$ )

(only 2 events shorter than $1 \mathrm{~s}$ ), a partial correlation between intensity and incoming direction (weaker events are preferably triggered at directions close to the satellite's equatorial plane). The angular distribution of these GRBs in the satellite rest frame reveals the pair of detectors GRBM2 and GRBM3 as the most efficient in triggering GRBs (we remind here that a valid GRB is recognized when at least 2 detectors trigger on the same event).

\section{GRB localization method}

The method we follow for the localization of a GRB source is based on the comparison between the relative intensities registered by the four GRBM detectors and the expected values for the same ratios, as derived from the instrument's response functions. For a given GRB, we identify the two most illuminated detectors and consider the expected ratios between the registered intensities for each possible incoming direction (in this preliminary phase spectral characteristics are derived from BATSE data). These ratios are then compared with the observed ones, and only those directions whose expected and observed values are compatible are classified as possible locations for the source. This procedure determines a first error box whose shape and dimensions are strongly dependent on the GRB intensity and incoming direction. The same comparison is carried out with different couples and/or combinations of detectors and energy ranges to provide the maximum information. Finally the various error boxes are overlapped to obtain the best localization of the source.

\section{The first localization of a GRB}

We have applied this method to GRB970517 (BATSE $\sharp 6235$ ). We chose this GRB for its characteristics of intensity, morphology and relative intensities registered in different detectors: it is a strong, sharp burst lasting about $5 \mathrm{~s}$, with approximately the same signal in detectors GRBM2 and GRBM3. In this preliminary attempt, we used BATSE data to derive its spectral properties (spectral index $\alpha=0.9$, assuming a power-law spectrum $\left.\propto E^{-\alpha}\right)$. As a preliminary determination of the

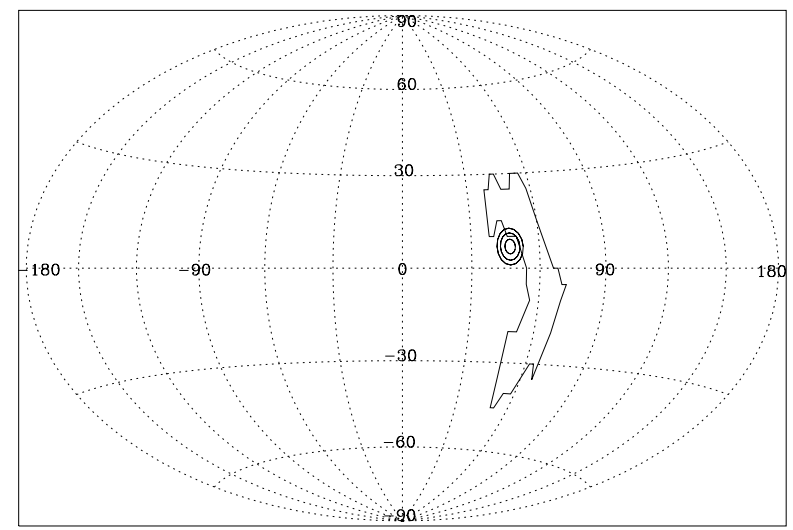

Fig. 2. The GRBM 90\% confidence level error box of GRB 970517. The coordinate system is centered on the axis of detector GRBM3. Also the BATSE 68\%, 90\% and $95 \%$ confidence radii (centered at $\theta=6.8^{\circ}, \phi=47^{\circ}$ ) are shown, as derived from the double-Fisher error distribution in Briggs et al. (1999)

GRB incoming direction we have only considered the ratio between the GRBM2 and GRBM3 intensities, whose dependence on the azimuth $\phi$ is shown in Fig. 1. The comparison between observed and expected ratio gives a strong selection of the possible values of $\phi$ as a function of the altitude $\theta$. The same criterion has been used to select a combination of detectors intensities giving a good selection of $\theta$ versus $\phi$. The ratio between the sum of detectors GRBM1 and GRBM4 and the sum of detectors GRBM2 and GRBM3 proved to give the best results. Both computations were carried out for the two GRBM spectral bands (GRBM band, 40-700 keV and AC band, 100-700 keV). The final error box is shown in Fig. 2 along with BATSE's position.

\section{Conclusions}

A first attempt to localize a GRB source with the GRBM has shown a good agreement with results obtained with BATSE. Further improvements of the method include the independent determination of the spectral index and its standardization for a quick look use. The authors wish to thank the BATSE team for making their data publicly available.

\section{References}

Amati L., Cinti M.N., Feroci M., et al., 1997, Proc. SPIE 3114, 176

Boella G., Butler R.C., Perola G.C., et al., 1997, A\&ASS 122, 299

Briggs M.S., Pendleton G.N., Kippen R.M., et al., 1999, astro-ph/9901111

Costa E., Frontera F., Dal Fiume D., et al., 1998, Adv. Sp. Res. 22, 1129

Feroci M., Frontera F., Costa E., et al., 1997, Proc. SPIE 3114, 186

Feroci M., et al., 1999 (this volume)

Fishman G., et al., 1985, Proc. 19th ICRC 3, 343

Frontera F., et al., 1997, A\&ASS 122, 357

Pamini M., et al., 1990, Il Nuovo Cimento 13C, 337 